\title{
Salivary proteins in health and disease
}

\author{
Dorota Kościelniak¹, Anna Jurczak¹, Agnieszka Zygmunt² and Wirginia Krzyściak²凶 \\ 1Department of Pediatric Dentistry, Institute of Dentistry, Jagiellonian University, Medical College, Cracow, Poland; ${ }^{2}$ Department of Medical \\ Diagnostics, Jagiellonian University, Medical College, Faculty of Pharmacy, Cracow, Poland
}

\begin{abstract}
Besides their structural catalytic and diverse regulatory functions, proteins are also precursors of many important biological compounds essential for normal functioning of humans. Many of these compounds may be used as markers for identification of specific pathological states. A comprehensive knowledge about the metabolism of salivary proteins and the mechanisms of action of their metabolites allowed the development of effective treatment for many disorders. However, it should not be forgotten that in some pathological conditions, these compounds not only could be involved in the pathogenesis but also could be used as tool in the prediction of many diseases. This paper is a review of the published literature on selected salivary proteins in the context of the physiological processes of the human body and chosen chronic disorders, such as diabetes, diabetic nephropathy, mucositis, oral mycoses and caries.
\end{abstract}

Key words: proline-rich proteins, $\beta$-defensins, oral cavity, diagnostics Received: 21 June, 20912; revised: 21 September, 2012; accepted: 24 October, 2012; available on-line: 16 November, 2012

\section{THE SIGNIFICANCE OF SELECTED SALIVARY PROTEINS AND THEIR METABOLITES IN PHYSIOLOGY AND PATHOLOGY}

The number of human proteins considerably exceeds the number of genes, which is connected with the phenomenon of alternative splicing and post-translation modifications occurring in the course of gene expression. This leads to the formation of proteins of various structures. It appears that even a few dozen proteins may be formed from one gene, and therefore the definition of the complete human proteome is a much more complicated task than genome definition. The proteomics research is currently focused on two areas: the first is connected with protein expression, and the second with their behavior in biological systems.

Salivary proteins play a significant role in protecting humans against infection. Their level in the oral cavity is subject to constant fluctuations which depend on numerous factors. Modification of the composition of salivary proteins is caused not only by pathological changes, but is also a result of numerous physiological states. Physical exercise, among others, may cause changes in the concentration of salivary proteins, such as $\beta$-defensins and LL-37. Fujimoto et al. (2011) observed that physical exercise leads to an increase in the level of $\beta$-defensin-2 and peptide LL-37 (Fig. 1). It is supposed that such a change may be connected with the release of a range of pro-inflammatory cytokines such as IL-1b or TNF- $\alpha$, which induce the release of antibacterial peptides. This process probably also involves catecholamines synthe- sized during an exercise; however, their role in that range has not been proved so far. Moreover, it was noted that the increase in the level of $\beta$-defensin- 2 and LL-37 was negatively correlated with cortisol concentration (Fig. 1). Glycocorticoids may probably decrease the expression of $\beta$-defensins in saliva, thus limiting their ability of counteracting oral cavity infections (Fujimoto et al., 2011). An increase in the concentration of LL-37 and HNP1-3 (human neutrophil peptide, HNP) after the physical exercise was demonstrated in the study by Allgrove et al. (2009). The change in the amount of these proteins results from the inflammatory state caused by an intense physical exercise. This involves an increase in the level of neutrophil granulocytes, from which antibacterial proteins are released, both in the blood and saliva (Fig. 1) (Allgrove et al., 2009).

The level of salivary proteins changes with age. Boi et al. (2011) demonstrated an elevated concentration of total peptides in the saliva of preterm infants. Nine months later, the level of salivary proteins decreased reaching values observed in babies born on time. Proteins detected in considerable amounts included small proline-rich protein (SPRR) and peptides belonging to the S100 protein family. The elevated level of two isoforms of SPRR protein is probably connected with the lack of epithelial transglutaminase in preterm infants. Also an increased level of cystatins was observed in the infants'saliva. It was noted that there were differences in the concentration of particular salivary peptides depending on the age. The level of alkaline proline-rich proteins increased during the maturation period (Boi et al., 2011). This increase may be a result of changes which occur in salivary glands, but also a diet influence is considered as one of the reasons. It was demonstrated that consumption of products rich in tannins may increase proline-rich protein (PRP) expression (Ann et al., 1997). Moreover differences in the level of particular PRP peptides were noticed. II-2 peptide was the last to reach a constant concentration in saliva, which proves a differentiated expression of genes encoding particular PRP proteins. A substantial drop in acidic PRP level was observed in children aged 6-9 years due to the replacement of deciduous teeth with permanent ones (Boi et al., 2009). A rapid adsorption of acidic PRP on the surface of teeth

e-mail: wirginiakrzysciak@cm-uj.krakow.pl

Abbreviations: GPCR, G-protein-coupled receptor; hLF1, human lactoferrin 1; HNP, human neutrophil peptide; HSC70, heat shock cognate protein 70; Ig, immunoglobulin; II-2, proline-rich peptide II-2; IL, interleukin; LPS, lipopolysaccharides; LL-37, cathelicidin; P-113, derivative of histatin-5; P-B, proline-rich peptide P-B; P-C, proline-rich peptide P-C; Phd1, derivative of $\beta$-defensin-1; Phd2, derivative of $\beta$-defensin-2; Phd3, derivative of $\beta$-defensin-3; PRP, proline-rich protein; S100, S100 calcium binding protein; SPRR, small proline-rich protein; STAT 3, signal transducer and activator of transcription 3; Th17, helper lymphocyte 17; TNF-a, tumor necrosis factor-a; TLR, Toll-like receptor. 


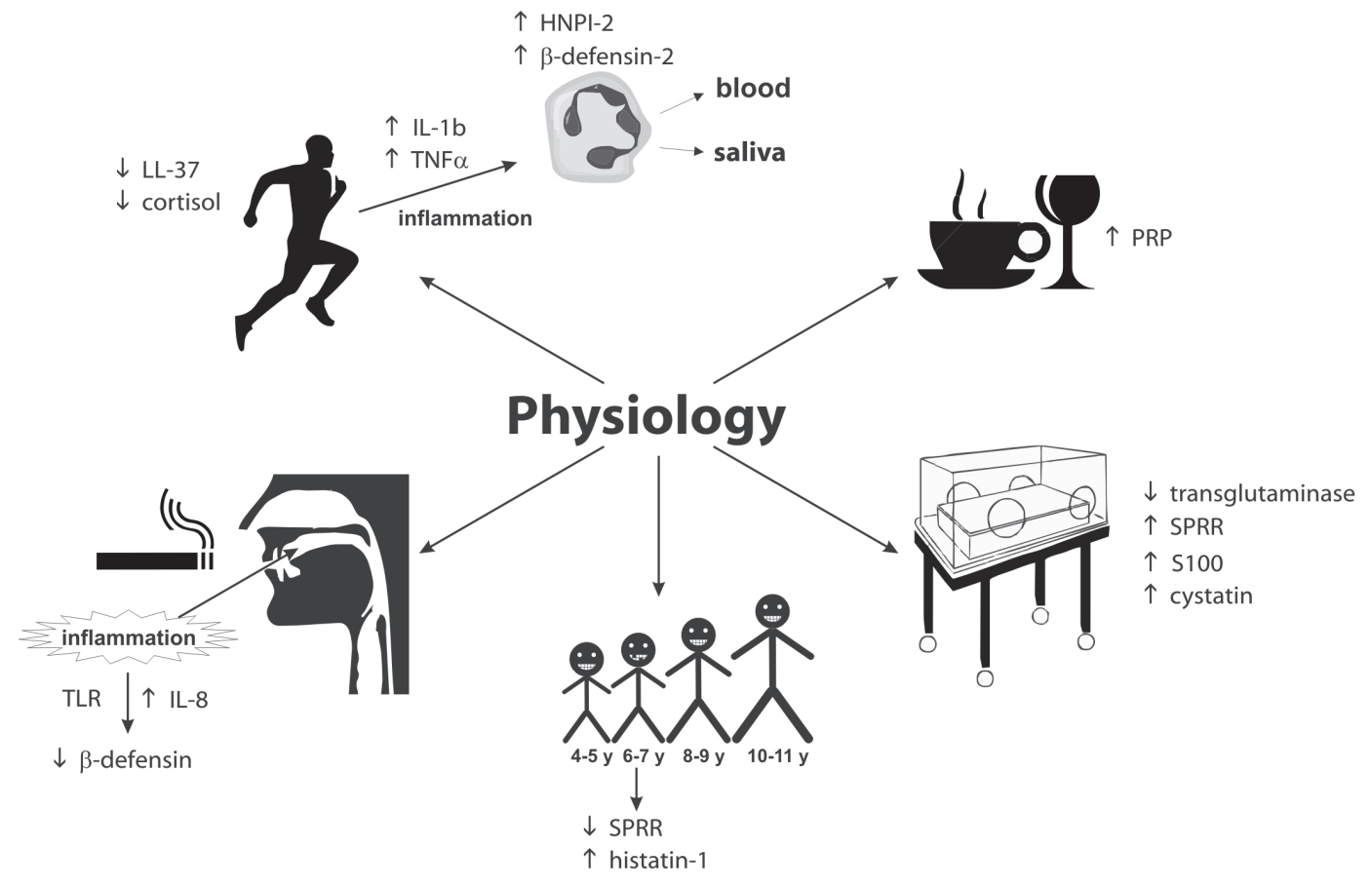

Figure 1. Selected salivary proteins in physiology.

and gums takes place during this process, decreasing their in level in saliva (García-Godoy \& Hicks, 2008). In the group of 3-5-year-old children, in turn, a higher level of histatin 1 was noted. This could be due to the eruption of deciduous teeth and the role of histatin 1 in the healing of wounds and lesions of the oral cavity tissues (Boi et al., 2009).

The level of antibacterial proteins in saliva is also influenced by substances which get to the oral cavity from, e.g. tobacco smoke. Changes in the production of IL-8 and $\beta$-defensin- 2 in epithelial cells of gums caused by tobacco components were assessed in a study by Eksomtramate et al. (2009). An increase in IL-8 expression by cigarette smoke was demonstrated, and the synthesis of $\beta$-defensins appeared to be lowered when compared to non-stimulated cells. Moreover, it was demonstrated that the activity of nicotine alone causes considerably lower effects, which suggests that the changes observed may be also influenced by other components of tobacco smoke (Eksomtramate et al., 2009). Mahanonda \& Pichyangkul (2007) demonstrated that induction of IL-8 and $\beta$-defensins is mediated by TLR receptors (Toll-like receptor) present in the oral cavity epithelium. They are activated by, among other factors, lipopolysaccharides (LPS) of bacterial cell membranes (Mahanonda et al., 2007). Lowering of the synthesis of $\beta$-defensins which have antibacterial properties leads to impaired ability of an organism to fight oral cavity pathogens. An increase in IL-8 concentration, in turn, potentiates the inflammatory state in periodontium diseases. It may be concluded that avoiding the intake of some substances may enhance the protection against pathogens occurring in the oral cavity (Fig. 1).

Various pathological states may be both a result and a reason of modification of salivary protein composition. One of diseases leading to changes in the level of antibacterial proteins in oral cavity is diabetes. Cabras et al. (2010) demonstrated differences in the concentration of the salivary proteins between healthy children and those with type 1 diabetes. The level of $\alpha$-defensins 1, 2 and 4, S100A9, the calcium binding protein, and small fragments of PC peptide were all more abundant in the diabetic group. It was also noted that the concentration of $\alpha$-defensins 1,2 and 4 in saliva strongly correlates with their level in the blood (Cabras et al., 2010). The concentration of these proteins was considerably elevated in patients with diabetic nephropathy, which is probably a result of an impaired efficiency of their removing by kidneys. Also the role of inflammatory state cannot be excluded, which involves an activation of neutrophils and an increased in release of $\alpha$-defensins stored in the granulations of neutrophil granulocytes (Flyvbjerg et al., 2008). The concentration of statherins, histatins 1 and 3 , and proline-rich peptide PB was considerably lowered in the examined group. Moreover, a decrease in the level of PC peptide originating from pre-secretory modification of PRP-1 protein was demonstrated. An increase in the concentration of fragments originating from PC peptide degradation occurs concurrently. An increased activity of glutamine endoprotease originating from microorganisms is observed in patients with diabetes, which explains an increase in the level of PC peptide fragments in saliva of the diseased subjects.

A decrease in the level of statherins and their derivatives was also noted in the examined group, which suggests lowered expression of genes for these peptides in patients suffering from diabetes. Similarly, the concentration of $\mathrm{PB}$ peptide belonging to the family of basic proline-rich proteins was decreased in the saliva of diabetic children. Moreover, a lowered concentration of histatin 1 , which is an adjunctive factor in wound healing, was noted in the examined group (Cabras et al., 2010). The ability of histatins to stimulate wound healing was confirmed in a study by Bolscher et al. (2009). A three-dimensional dermal model obtained using tissue engineering methods was used for this purpose. It was observed that histatin leads to tissue regeneration not by increasing cell proliferation, but by enhancing their mobility. Histatins probably affect skin cells via G-protein- 
coupled receptor (GPCR) passing the signal stimulating migration.

Moreover, it was demonstrated that peptide structure influences histatin properties. Protein cyclization increases the cell migration stimulatory activity 1000-fold (Bolscher et al., 2009). Blaauboer et al. (2010) demonstrated that histatin 2 intensifies migration of epithelial cells and fibroblasts, which enhances regeneration of damaged tissue without inflammatory state or fibrosis. Histatin 2 demonstrates an enantiomer-dependent effect on the excited cells (Blaauboer et al., 2010). Fujigaki et al. (2009) demonstrated that histatin 3 may increase proliferation of gum fibroblasts by interaction with peptides related to heat shock cognate proteins 70 (HSC70). HSC70 proteins form a complex with a cell cycle regulator, which leads to transition of the cells from the G1 to S phase and accelerates their proliferation (Fujigaki et al., 2009). Histatins may in future play an important role in wound healing therapy owing to their lack of cytotoxicity at concentrations of at least $100 \mu \mathrm{g} / \mathrm{ml}$, metabolic stability and small size, which facilitates penetration through skin surface (Bolscher et al., 2009). The process of tissue regeneration in diabetics seems to be impaired, thus the therapy with histatin 1 which stimulates regeneration of damaged tissues, may be a therapeutic concept in the future.

Statherins, histatins and PB peptide play a significant role in tooth tissue protection against caries, therefore lowering of their concentration in the saliva of children suffering from type 1 diabetes may favor pathological changes on tooth enamel (Cabras et al., 2010). Lowered concentration of histatins is partly a result of their forming complexes with other proteins, such as mucins, PRP, statherins or amylase. The major cause of protein binding are hydrophobic interactions, albeit hydrogen bonds also contribute in this process (Iontcheva et al., 1997). However, the main factor leading to a decrease is their proteolytic degradation resulting in the histatin level of the stable derivatives formation (Bosch et al., 2009). An important source of proteolytic enzymes are microorganisms of the oral cavity. It has been demonstrated that arginine-dependent gingipains produced by Porphyromonas gingivalis lead to degradation of histatin 5 (Baehni et al., 2001). Histatins are highly susceptible to proteolysis due to their high content of lysine and arginine residues (Alagl et. wsp., 2006). The decomposition of histatins in diabetics seems however to be balanced, which is proved by a lack of a considerable increase of the concentration of histatin derivatives when compared to their amount in healthy people. An increased level of S100A9 protein coming from neutrophil cytoplasm was also noted in the examined group. S100A9 is a part of the calprotectin complex, which is upregulated in response to LPS derived from the invading microorganisms (Akamatsu et al., 2009). Those authors demonstrated an increased phosphorylation, what was probably connected to an adaptation of neutrophil granulocytes cytoplasm to exocytosis (Cabras et al., 2010).

The above studies suggest that determination of the level of antibacterial proteins such as $\alpha$-defensins, statherins or histatins which are present in the saliva of patients with type 1 diabetes could be a useful tool for the detection and prevention of its complications.

The salivary protein composition may also change in the course of hyper- $\operatorname{IgE}$ syndrome which is characterized by an increased concentration of $\mathrm{IgE}$ and recurrent infections of mucous membranes and skin. A decrease in the level of proteins of anti-fungal activity such as $\beta$-defensins- 2 and histatin 1 in hyper-IgE syndrome was demonstrated in a study by Baker et al. (2011). They observed that incubation of epithelial cells of the oral cavity with IL-17 leads to an increase in histatin 1 level. This suggests a role of Th17 lymphocytes in the expression of antibacterial proteins in the saliva. Lowered level of Th17 or disturbed signalization of STAT 3 protein may probably cause a lowered level of histatin and $\beta$-defensin- 2 . Also, different levels which of these proteins were demonstrated among patients in the control group (Baker et al., 2011). Lowered concentration of $\beta$-defensin- 2 and histatin 1 is a significant factor promoting bacterial and fungal infections in the course of hyper-IgE syndrome.

Also Sjögren's syndrome is accompanied by changes in salivary proteins. The aim of a study by Gunduz et al. (2009) was to determine the level of $\beta$-defensin -1 and -2 in the saliva of those suffering from the disease. Expression of the antibacterial proteins was decreased in the diseased subjects, and the reduction of $\beta$-defensin- 2 was higher than that of $\beta$-defensin-1. Infiltration of salivary glands by lymphocytes occurs in Sjögren's syndrome. It was demonstrated that the decrease in the level of $\beta$-defensins in salivary glands of the diseased may occur just before the infiltration by the cells of the lymphatic system. The deficiency of $\beta$-defensins may thus be one of the factors leading to development of Sjögren's syndrome (Gunduz et al., 2009). By analyzing the saliva proteome of the diseased, the researchers found a different pattern than in physiological conditions, and thus these proteins may become biomarkers of a specified pathological state.

One of the pathological processes accompanied by changes in salivary proteins is caries. Amado et al. (2010) examined the concentration of PRP proteins, statherins and histatins in the saliva of subjects affected with caries and a control group without clinical symptoms. Salivary peptides, including PRP proteins, statherins and histatins, can form complexes protecting them from proteolysis. Such a form facilitates binding to the enamel surface and formation of pellicula which prevents demineralization of teeth (Iontcheva et al., 1997). It has been demonstrated that the presence of pellicula decreases attachment of caries-causing bacteria to the tooth surface. However, formation of a more stable layer is needed in order to ensure enamel protection against the loss of calcium phosphate salts. This process involves transglutaminase, an enzyme released by epithelial cells of the oral cavity which enables formation of covalent bonds between salivary proteins (Grogan et al., 2000). It has been demonstrated that the ratio concentration of proteins attached to connected with the enamel hydroxyapatite to those present in free form is considerably higher in healthy people. Such a relationship concerns statherins, histatin 1, PRP-1 and PRP-3 proteins. Attachment of these proteins with hydroxyapatite enables better protection of teeth against acids, providing proper remineralization of the enamel. Moreover, a higher total amount of peptides was demonstrated in the saliva of people without caries. Also lowered susceptibility of histatin 5 connected to hydroxyapatite to proteolytic a degradation was noted. In turn, in the group of people with caries, an increase in the level of histatin derivatives and a decrease in the concentration of the parent forms was observed, which favored demineralization process. Binding of salivary proteins with hydroxyapatite is one of the mechanisms of enamel protection against caries development.

The antibacterial activity of two salivary peptides: fragment of active lactoferrin domain (hLF1-11- human lactoferrin 1-11) and P-113, which is 12-amino acid derivative of histatin 5 , was assessed in a study by Huang 
et al. (2011). Streptococcus mutans strains were the most susceptible to the antibacterial activity of the mentioned peptides, while the growth of $S$. gordonii and $S$. sanguis strains was inhibited by higher levels of hLF1-11 and P-113 peptides. The application of electron microscopy showed that the peptides penetrate the bacterial cell without causing its lysis. hLF1-11 and P-113 accumulate in the microorganism's cytoplasm, and their bactericidal activity is probably based on interactions with the DNA of the host. Due to its high content of the basic amino acid arginine and the ability to form hydrogen bonds, hLF1-11 may bind to the bacterial cell surface and then undergo spontaneous internalization. The N-terminal part of the peptid containing four arginine residues contributes to the binding of hLF1-11 to DNA. In has been demonstrated that the interaction of lactoferrin with DNA involves electrostatic interactions between positively charged amino acids with the negatively charged deoxyribonucleic acid (De Boer et al., 1997). Similar situation may also concern the P-113 peptide which contains lysine residues. Details concerning the interaction of P-113 with DNA have not been, however, fully explained (Huang et al., 2011). The above reports may lead in the future to the construction of high-molecular-mass compounds based on peptides derived from lactoferrin and histatin 5 . They could be used in caries prevention.

Histatin plays a significant role in tooth enamel protection, therefore decomposition of this protein is an unfavorable phenomenon. The influence of histatin 1 binding with teeth hydroxyapatite on its proteolytic degradation was examined in a study by Goldberg et al. (2011). The enzymes decomposing histatin 1 may be derived from pathogenic bacteria present in the oral cavity, mainly Porphyromonas gingivalis and Bacteroides forsythus. An ability of human serine proteases to degrade histatin 1 has also been demonstrated. The C-terminal part of histatin, rich in arginines and lysines, is the most likely to undergo proteolysis. Histatins, both unbound and attached to hydroxyapatite, demonstrate a similar degradation pattern forming eight fragments, including one responsible for stimulation of wound healing. The presence of the peptide enhancing reconstruction of damaged tissue may be of significant importance in the treatment of periodontium diseases. Although histatin can be degraded both in the free and bound form, the proteolysis of the protein connected to hydroxyapatite is five-fold slower. This is probably due to a decreased accessability of proteolytic enzymes to the bound histatins (Goldberg et al., 2011). The results of the above studies may be a basis for the synthesis of molecules to be used in the therapy of caries and periodontium diseases.

A next group of proteins playing an important role in maintaining gum health are defensins. Ackermann et al. (2010) examined the ability of human $\alpha$ - and $\beta$-defensins to inhibit the pro-inflammatory cytokines of synthesis and to increase production of class $\operatorname{IgG}$ antibodies as a response to infection with Porphyromonas gingivalis. For this purpose, an experiment on mice was conducted, in which the mice were given recombined adhesins derived from that bacteria: hemagglutinin B and fibrillin. It was demonstrated that Porphyromonas gingivalis antigens mediated by lymphocytes Th17 stimulate the synthesis of pro-inflammatory cytokine IL-6 and chemokines. The response of the immunological system was lower in the presence of human $\alpha$ - and $\beta$-defensins (Ackermann et al., 2010). Thanks to the proximity of the site of $\beta$-defensins'synthesis to the area of bacteria activity, the peptides could bind the recombined bacterial adhes- ins before their presentation by the cells of the immunological system. Formation of the protein-antigen complex probably proceeds via electrostatic interactions. The $\beta$-defensin binding with the bacterial antigen changes its conformation which disturb signalization inside dendritic cells of bone marrow and decreases the concentration of pro-inflammatory cytokines (Belanger et al., 2008). The result of the study suggests the possibility of application of defensins for combating infections caused by $P$. gingivalis.

The contribution of $P$. gingivalis bacteria in the induction of changes in periodontal tissues is the subject of research of Jan Potempa and his team. Their study is focused on the role of gingipains, enzymes produced by $P$. gingivalis, in the pathogenesis of periodontium diseases. Gingipains are cysteine, serine proteases preferentially cleaving peptide bounds following cysteines, serines or arginines. Those researchers have demonstrated that cysteine proteases can stimulate apoptosis of gum epithelial cells which leads to the destruction of periodontium tissues (Benakanakere et al., 2009). Gingipains induce cell death by disturbing the interaction of adhesive molecules connecting epithelial cells of the gum with proteins of the extracellular matrix (Guo et al., 2010). As a response to the colonization of teeth by bacteria, serine proteases are released from granulations of neutrophil granulocytes: elastase and protease 3 , which not only destroy the microorganisms responsible for the tooth decay, but also induce the inflammatory state and damage the periodontium tissues. Limitation of these processes is ensured by the presence of leukocyte protease inhibitor elafin which inhibits the neutrophil elastase and protease 3. It's synthesis is induced in inflamed epithelial cells. C-terminal fragment of this protein contains four disulphide bridges and is the site of serine protease binding, being responsible concurrently for their deactivation (Hirose et al., 1999). The N-terminal domain of elafin is bound to extracellular matrix. Besides inhibiting serine proteases, elafin also demonstrates an antimicrobial activity by interacting electrostatically with bacterial LPS (Alam et al., 2012). It was demonstrated that gingipains released by $P$. gingivalis inactivate the inhibitors of elastase and protease 3 , which creates conditions for the development of periodontium inflammatory state (Eick et al., 2012).

Beta defensins also contribute to changes occurring in periodontal tissues. An increase in prostaglandin $\mathrm{E}_{2}$ level is observed in periodontium diseases due to inflammatory state formation. Chotjumlong et al. (2010) demonstrated that $\beta$-defensin-3 present in guts epithelial cells may interact in a paracrine manner with the fibroblast layer below them. An increase in prostaglandin $\mathrm{E}_{2}$ level is then observed. It was noted that its induction is mediated by cyclooxygenase- 2 . $\beta$-defensin-3 probably also influences the expression of phospholipase A2 taking part in arachidonic acid metabolism. These results confirm the considerable role of $\beta$-defensins in formation of inflammatory state in periodontium diseases.

Another disease of the oral cavity in which saliva proteins may find application therapeutics is mycosis caused by Candida albicans. Protective action of histatin 5 in oral cavity colonization by yeast-like fungi was the subject of a study by Fidel et al. (2010). Mouse tongue was used as a research model because of the similarity of processes occurring in their mocous membrane with those in the human oral cavity. It was observed that an increase in histatin 5 concentration is accompanied by a decrease in the density of $C$. albicans colonies. Numerous C. albicans colonies may, however, lead to histatin 5 degradation, which was demonstrated by inhibiting the histatin 
Table 1. The changes of selected salivary proteins in physiology/pathology.

\begin{tabular}{|c|c|c|c|}
\hline Physiology/pathology & Protein & Level & Literature \\
\hline \multirow[t]{3}{*}{ Physical exercise } & $a, \beta$-defensins & $\uparrow$ & Fujimoto et al. (2011) \\
\hline & LL-37 & $\uparrow$ & Allgrove et al. (2009) \\
\hline & HNP 1-3 & $\uparrow$ & \\
\hline \multirow[t]{2}{*}{ Preterm infants } & SPRR & $\uparrow$ & Boi et al. (2011) \\
\hline & $\mathrm{S} 100$ & $\uparrow$ & \\
\hline Infants & Cystatins & $\uparrow$ & \\
\hline Diet rich in tannins & PRP & $\uparrow$ & Ann et al. (1997) \\
\hline Maturation & PRP & $\uparrow$ & García-Godoy \& Hicks (2008) \\
\hline $3-5$ years old & His-1 & $\uparrow$ & Boi et al. (2009) \\
\hline \multirow[t]{2}{*}{ Smoking } & IL-8 & $\uparrow$ & Eksomtramate et al. (2009) \\
\hline & $\beta$-defensins & $\downarrow$ & Mahanonda et al. (2007) \\
\hline \multirow[t]{7}{*}{ Diabetes type 1} & His-5 & $\uparrow / \downarrow$ & Baehni et al. (2001) \\
\hline & a-defensine 1, 2, 4 & $\uparrow$ & \\
\hline & S100A9 & $\uparrow$ & Akamatsu et al. (2009) \\
\hline & PC & $\uparrow$ & \\
\hline & His 1-3 & $\downarrow$ & Cabras et al. (2010) \\
\hline & Staterins & $\downarrow$ & \\
\hline & PB & $\downarrow$ & \\
\hline \multirow[t]{3}{*}{ Hyper-lgE syndrome } & $\lg E$ & $\uparrow$ & Baker et al. (2011) \\
\hline & $\beta$-defensin-2 & $\downarrow$ & \\
\hline & His-1 & $\downarrow$ & \\
\hline \multirow[t]{2}{*}{ Sjögren's syndrome } & $\beta$-defensin-2 & $\downarrow \downarrow$ & Gunduz et al. (2009) \\
\hline & $\beta$-defensin-1 & $\downarrow$ & \\
\hline \multirow[t]{8}{*}{ Caries } & Staterins & $\downarrow$ & Belanger et al. (2008) \\
\hline & His-1 & $\downarrow$ & Goldberg et al. (2011) \\
\hline & PRP-1 & $\uparrow$ & Huang et al. (2011) \\
\hline & PRP-3 & $\uparrow$ & Dietrich et al. (2009) \\
\hline & His-5 & $\downarrow$ & Bal et al. (2011) \\
\hline & IL-6 & $\uparrow$ & \\
\hline & Chemokines & $\uparrow$ & Chotjumlong et al. (2010) \\
\hline & $\mathrm{PGE}_{2}$ & $\uparrow$ & Harriott et al. (2008) \\
\hline
\end{tabular}

bactericidal activity. In addition, it was proved that the antifungal activity of histatin is considerable and reaches maximum only after 30 minutes (Fidel et al., 2010). Due to the ability of histatins to protect against the pathogenic C. albicans activity, and a lack of a toxic impact on host cells, these proteins may become a therapeutic used in prevention and treatment of oral cavity candidiasis.

$\beta$-defensins also demonstrate an antifungal activity. Their derivatives containing the C-terminal fragment of the parent peptide, such as Phd1, Phd2 and Phd3, have similar properties. Krishnakumari et al. (2009) demonstrated that the minimum fungicidal concentration was lower for $\beta$-defensins, which suggests about their higher activity towards $C$. albicans. Derivatives of $\beta$-defensins demonstrated in turn higher fungicidal abilities in the presence of various concentrations of $\mathrm{NaCl}, \mathrm{CaCl}_{2}$ or $\mathrm{MgCl}_{2}$. Metabolic inhibitors that limit ATP accessibility did not negatively affect the antifungal properties of Phd1, Phd2 and Phd3, but considerably decreased the activity of $\beta$-defensins. These results suggest that the mechanism of $C$. albicans combating by of $\beta$-defensin derivatives are not ATP-dependent. It was noted that $\beta$-defensins and their analogues containing the C-terminal fragment lead to permeabilization of $C$. albicans cell membrane. The highest activity in permeabilization was demonstrated with $\beta$-defensin-3 and its derivative Phd3 (Krishnakumari et al., 2009). Thus, the C-terminal fragments of $\beta$-defensins may be a relatively safe and effective approach to the therapy of oral cavity candidiasis in the future.

Some oral cavity peptides demonstrating antibacterial activity may be a therapeutic mean only after their modification. The antibacterial activity of a derivative of HNP-1 peptide belonging to $\alpha$-defensin family was determined in a study by Harriott et al. (2008). The factor limiting the HNP-1 application in infection therapy is the presence of three disulphide bridges responsible for the antibacterial properties and formation of a suitable spatial structure. However, such a conformation cannot be obtained by chemical synthesis (Antonyraj et al., 2000). 
Bactericidal properties are also attributed to the C-terminal fragment of that protein, which was also the basis of obtaining HNP-1 analogues without bonds between cysteine residues. The widest spectrum of antibacterial activity was observed for a peptide named $2 \mathrm{Abz}^{23} \mathrm{~S}^{29}$, which was effective towards Escherichia coli, Staphylococcus aureus, Enterococcus faecalis and Streptococcus mutans strains. A slightly stronger activity towards the latter bacteria was demonstrated by another peptide $-2 \mathrm{Abz}^{14} \mathrm{~S}^{29}$. The antibacterial activity of that derivative may result from its chemical modification consisting in the substitution of glycine in position 23 by a 2-aminobenzoyl derivative. Moreover, the bactericidal properties of HNP-1 derivatives are connected with their cationic character. The isoelectric points of the HNP-1 analogues and of the parent peptide were higher for the derivatives showing antibacterial properties than for those without bactericidal activity. These data suggest that a higher positive charge increases the antibacterial activity of HNP-1 derivatives (Harriott et al., 2008). Due to their demonstrated antibacterial properties, HNP-1 derivatives without disulphide bridges may be a starting point for the synthesis of peptides applicable in the therapy of oral cavity infections.

Salivary proteins also contribute to changes in the oral cavity involving the sensation of tanginess resulting from consumption of various foods. This is a result of the peptides binding the polyphenols contained in the food. Glycosylated PRPs are, to a high degree, responsible for the feeling of the dryness in mouth after consumption of food rich in tannins. PRPs play a role of moistening factors decreasing the friction in the oral cavity. Their binding with polyphenols and further precipitation lead to a loss of these protective properties which stimulates tanginess. Cabane et al. (2008) demonstrated that glycosylated PRPs interact with tannins which leads to the formation of precipitating complexes. The aggregation was stronger in the case of more polymerized polyphenols. Flavan3-ol polymers are present, e.g., in dry red wine, and thus its consumption induces stronger feeling of dryness in the oral cavity when compared to other foods containing non-polymerized polyphenols. The results obtained were compared with the precipitation degree of nonglycosylated PRP. The aggregation was stronger in that case and led to phase separation. It is thus suggested that glycosylation may limit precipitation of protein-polyphenol complexes (Cabane et al., 2008). In turn, Freitas et al. (2012) it was demonstrated that after binding with polyphenols, statherins and acidic PRP were the first to precipitate. Glycosylated PRP precipitated next, while basic PRP required an application of considerable concentrations of tannins. These results prove a high affinity of acidic PRP and statherins to polyphenols. In addition, it was demonstrated that protein aggregation is higher in acidic saliva which does not contain amylase and mucins. This proves a role of these proteins in inhibition of precipitation (Freitas et al., 2012).

In addition to polyphenols binding, salivary proteins may also form complexes with metal ions. Dietrich et al. (2009) demonstrated that PRPs are able to bind $\mathrm{Cu}^{2+}$ ions by electrostatic interaction with the negatively charged residues of asparagine and glutamate. The copper ion binding may induce the feeling of dryness in the mouth. The mechanism is similar to that describe earlier for the PRP interaction with polyphenols, since in both cases precipitation of PRP proteins occurs, which decreases the saliva viscosity. It was demonstrated that other low-molecular-mass proteins such as mucins and amylase can also bind copper ions (Dietrich et al., 2009).
An ability of histatin 5 and its two analogues to bind nickel (II) ions was assessed by Bal et al. (2011). This element is a strong allergen present in food and drinks and until recently in orthodontic braces. It may cause an allergic reaction in the sensitized. The main nickel-binding protein is albumin present in the serum and body fluids. It was noted, however, that $\mathrm{Ni}^{2+}$ attachment to that protein is five-fold weaker than to histatin 5. It was demonstrated that nickel binding to histatin molecule involves its $\mathrm{N}$-terminal fragment (Bal et al., 2011). Histatin 5 is thus a significant factor determining nickel toxicity and its allergen activity.

Salivary proteins, due to the wide spectrum of their antimicrobial activity, play a considerable role in ensuring the proper functioning of the human organism. As presented in this paper, selected salivary proteins and their derivatives exert many important functions in the organism under normal physiological conditions. Moreover, many of these compounds may be used as markers for identification of specific pathological states (Table 1). In future, peptides isolated from the oral cavity may become a valuable tool in the diagnostics and therapy of numerous pathological processes.

\section{REFERENCES}

Ackermann A, Bélanger M, Brogden K, Burnell K, Cavanaugh J, Kohlgraf K, Lu X, Progulske-Fox A, Xie H (2010) Defensins attenuate cytokine responses yet enhance antibody responses to Porphyromonas gingivalis adhesins in mice. Future Microbiol 5: 115.

Akamatsu T, Azlina A, Hasegawa T, Hiroshima Y, Hosoi K, Javkhlan P, Kido J, Nagata T, Yao C (2009). Induction of calprotein mRNAs lipopolysaccharide in the salivary glans of mice. $J$ Med Invest 56: 287-289.

Alagl A, Helmerhost E, Oppenheim F, Siqueira W (2006) Oral fluid proteolytic effects on histatin-5 structure and function. Arch Oral Biol 51: 1061-1070.

Alam S, Henriksen P, Newby D (2012) Role of the endogenous elastase inhibitor, elafin, in cardiovascular injury. From epithelium to endothelium. Biochem Pharmacol 83: 695-704.

Allgrove J, Davison G, Gleeson M (2009) Salivary antimicrobial peptides (LL-37 and alpha-defensins HNP1-3), antimicrobial and IgA responses to prolonged exercise. Eur J Appl Physiol 106: 277-284.

Amado F, Lobo MJ, Domingues P, Duarte JA, Vitorino R (2010) Salivary peptidomics. Expert Rev Proteomics 7: 709-721.

Antonyraj K, Karunakaran T, Raj P (2000) Large-scale synthesis and functional elements for the antimicrobial activity of defensins. Biochem J 347: 633-641.

Baker O, Conti H, Edgerton M, Freeman A, Gaffen S, Holland S, Jang W, Li R (2011) New mechanism of oral immunity to mucosal candidiasis in hyper-IgE syndrome. Mucosal Immunol 4: 448-455.

Bal W, Bonna A, Goch G, Kurowska E (2011) Salivary histatin 5, a physiologically relevant ligand for $\mathrm{Ni}(\mathrm{II})$ ions. I Inorg Biochem 105: 1220-1225.

Belanger M, Brogden K, Burnell K, Cavanaugh J, Dawson D, Dietrich D, Eastman C, Hansen C, Guthmiller J, Johnson G, Joly S, Kohlgraf K, Kurago Z, Pingel L, Progulske-Fox A, Srikantha R, Xiao X (2008) Human b-defensin 3 binds to hemagglutinin B (rHagB), a non-fimbrial adhesin from Porphyromonas gingivalis, and attenuates a pro- inflammatory cytokine response. Immunol Cell Biol 86: 643-649.

Benakanakere M, Galicia J, Garcia C, Kinane D, Potempa J, Stathopoulou P (2009) Porphyromonas gingivalis induce apoptosis in human gingival epithelial cells through a gingipain-dependent mechanism. BMC Microbiology 9: 107.

Bolscher JG, Adão R, Nazmi K, van den Keybus PA, van 't Hof W, Nieuw Amerongen AV, Bastos M, Veerman EC (2009) Bactericidal activity of LFchimera is stronger and less sensitive to ionic strength than its constituent lactoferricin and lactoferrampin peptides. Biochimie 91: 123-132.

Cabane B, Pascal C, Poncet-Legrand C, Vernhet A (2008) Aggregation of a proline-rich protein induced by epigallocatechin gallate and condensed tannins: effect of protein glycosylation. I Agric Food Chem 56: $6724-6732$.

Cabras T, Pisano E, Mastinu A, Denotti G, Pusceddu PP, Inzitari R, Fanali C, Nemolato S, Castagnola M, Messana I (2010) Alterations of the salivary secretory peptidome profile in children affected by type 1 diabetes. Mol Cell Proteomics 9: 2099-2108. 
Chotjumlong P, Khongkhunthian S, Krisanaprakornkit S, Ongchai S, Reutrakul V (2010) Human $\beta$-defensin-3 up-regulates cyclooxygenase- 2 expression and prostaglandyn E2 synthesis In human gingival fibroblasts. I Periodont Res 45: 464-470.

De Boer H, Geerts M, Mericskay M, Nuijens J, Van Berkel P, Van Veen H (1997) N-terminal stretch Arg2, Arg3, Arg4 and Arg5 of human lactoferrin is essential for binding to heparin, bacterial lipopolysaccharide, human lysozyme and DNA. Biochem J 328: 145-151.

Dietrich A, Duncan S, Eigel W, Hong J, Mallikarjunan K, O’Keefe S (2009) Interaction of copper and human salivary proteins. J Agric Food Chem 57: 6967-6975.

Eick S, Guentsch A, Kantyka T, Laugisch O, Pfister W, Potempa J, Schacht M, Sculean A, Sroka A, Stennicke H (2012) Periodontal pathogens affect the level of protease inhibitors in gingival crevicular fluid. Mol Oral Microbiol 27: 45-56.

Fidel P Jr, Hackett W, Jabra-Rizk M, Peters B, Scheper M, Shaye S, Zhu J (2010) Protection of the oral mucosa by salivary histatin-5 against candida albicans in an ex vivo murine model of oral infection. FEMS Yeast Res 10: 597-604.

Freitas V, Mateus N, Soares S, Sousa A (2012) Effect of condensed tannins addition on the astringency of red wines. Chem Senses 37: 191-198.

Fujimoto S, Katsura Y, Orita K., Shin-ya U, Usui T, Yoshikawa T, Yoshimura M (2011) Changes in salivary antimicrobial peptides, immunoglobulin A and cortisol after prolonged strenuous exercise. Eur J Appl Physiol 111: 2005-2014.

Goldberg H.A, McDonald E, Mendes F.M, Tabbara N, Siqueira W (2011) Histatin 1 resists proteolytic degradation when adsorbed to hydroxyapatite. J Dent 90: 268-272.

Grogan J, Lendenmann U, Oppenheim F, (2000) Saliva and dental pellicle - a review. Adv Dent Res 14: 22-28.
Gunduz M, Kaneda Y, Katase N, Matsubara M, Mizukawa N, Nagatsuka H, Sawaki K, Takagi S, Yamaai T, Yamachika E, Yamanishi Y (2009) Localization of antimicrobial peptides human $\beta$-defensins in minor salivary glans with Sjögren's syndrome. Eur J Oral Sci 117: 506-510.

Guo Y, Nguyen K, Potempa J (2010) Dichotomy of gingipains action as virulence factors: from cleaving substrates with the precision of a surgeon's knife to a meat chopper-like brutal degradation of proteins. Periodontology 2000 54: 15-44.

Harriott P, Greer B, Lockhart D, Lundy F, Nelson J, Marley J (2008) Antimicrobial activity of truncated $\alpha$-defensin (human neutrophil peptide (HNP)-1) analogues without disulphide bridges. Mol Immunol 45: 190-193.

Hirose S. Schalkwijk J, Wiedow O (1999) The trappin gene family: proteins defined by an $\mathrm{N}$-terminal transglutaminase substrate domain and a C-terminal four-disulphide core. Biochem J 340: 569-577.

Huang X, Huo L, Gu L, Ling J, Liu H, Peng Z, Zhang K (2011) Antimicrobial and DNA-binding activities of the peptide fragments of human lactoferrin and histatin 5 against Streptococcus mutans. Arch Oral Biol 56: 869-876.

Iontcheva I, Oppenheim FG, Troxler RF (1997) Human salivary mucin MG1 selectively forms heterotypic complexes with amylase, prolinerich proteins, statherin, and histatins. J Dent Res 76: 734-743.

Krishnakumari V, Nagaraj R, Rangaraj N (2009) Antifungal activities of human beta-defensins HBD-1 to HBD-3 and their C-terminal analogs Phd1 to Phd3. Antimicrob Agents Chem 53: 256-260.

Mahanonda R, Pichyangkul S (2007) Toll-like receptors and their role in periodontal health and disease. Periodontology 43: 41-55. 